\title{
SEARCHING THE BEGINNING OF THE CLIMB TO GET THE MOST RELIABLE DIFFICULTY INDEX
}

\author{
Michat Wróbel \\ Institute of Computational Intelligence, Czestochowa University of Technology \\ Czestochowa, Poland \\ michal.wrobel@iisi.pcz.pl
}

Received: 1 December 2018; Accepted: 4 March 2019

\begin{abstract}
The climb is the steep fragment of the road. In road cycling, climbs should be categorized by the effort necessary to overcome it. Two values have the greatest influence on a difficulty of the climb: its average slope (rise/run ratio) and length. A simple approach to describing a climb's difficulty is multiplication slope squared and run. In this paper, the beginning of the climb is mathematically established to get the most reliable difficulty index. Moreover, it is presented a special case where the value of difficulty index does not depend on the location of the beginning point.
\end{abstract}

MSC 2010: 26A99, 00A79

Keywords: road cycling, altitude profile, difficulty index

\section{Introduction}

An important issue in road cycling is overcoming steep fragments of the course. In [1] it is said, that the mountain passes (besides the time trials) have a major impact on the final overall standings. Steep fragments of the roads, many times ended by a mountain pass, in cycling are usually called climbs. This term is used, for example, in [2] and [3].

It is necessary to describe and classify climbs, for instance, to establish a category of a mountain pass. As mentioned in [1], two values have the greatest impact on a climb's difficulty: its length and slope.

The slope is a rise/run ratio, usually given in percent. Rise (in this paper denoted by $h$ ) is the difference between the altitude of the end of the road and the altitude of the beginning of the road. Run (denoted by $r$ ) is just the horizontal length of the road (see Fig. 1). Therefore, the average slope (denoted by $m$ ) of the climb is expressed by the formula

$$
m=\frac{h}{r}
$$


Similarly, in [4] a climbing index is defined, but that value describes the difficulty of the whole race (the total climbed altitude divided by the total distance).

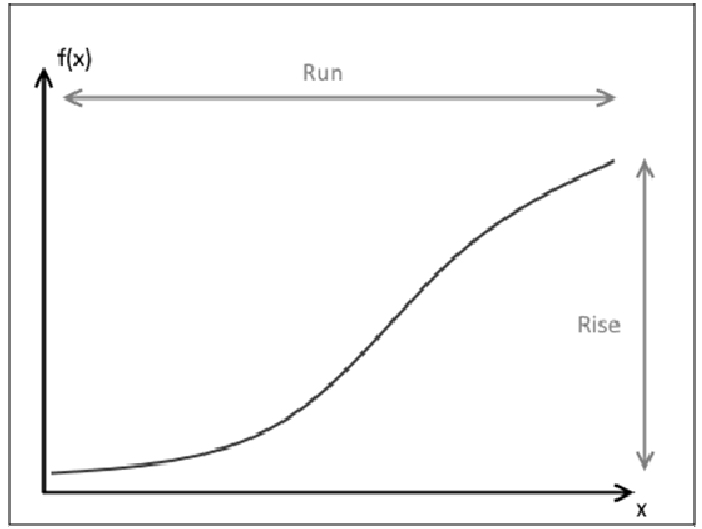

Fig. 1. Rise and run of the climb

It is good to have one value describing the difficulty of the climb - we would like to compare different climbs with each other. This value should depend both: run and slope. One approach to calculating climb difficulty is presented in [5] by the formula

$$
t=m^{2} r=\frac{h^{2}}{r} .
$$

Similar formulas are used in some websites containing descriptions of various climbs. This formula is quite simple (even for non-scientific use) and includes both needed values. Simpler formula $t=m r$ would be incorrect, because it depends only on rise $\left(m r=\frac{h}{r} r=h\right)$.

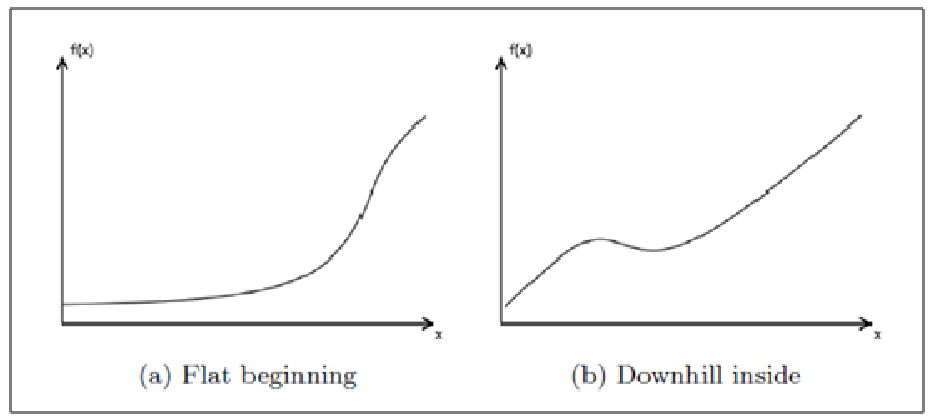

Fig. 2. Climbs with not obvious beginnings

The important issue is the delimitation of the climb from the whole road profile. The end of the climb is usually intuitively located close to the peak, but establishing the beginning is usually not obvious. Often, the initial part of the road is flat and the slope increases bit by bit (Fig. 2a). In this case, longer climb has got 
a greater length, but a smaller slope. Another example is that sometimes the road rises and then falls a little again (Fig. 2b). In this case, the first climb may be included or not.

In this paper, we are looking for a point where we should establish the beginning of the climb to get the most reliable difficulty index $t$.

\section{Definitions}

Let us observe that every road profile can be identified with the graph of a certain function $f$ where the horizontal distance of the point from beginning of the road turns into its altitude. An example of this graph is presented in [2], where it is called an altitude profile. Of course, the formula of $f(x)$ is unknown (it comes from area measurements), but we may assume that it is continuous and differentiable.

Let $x_{m}$ be an argument where the function $f$ attains its local maximum. We are interested in determining the difficulty of a part of the road ahead of this point. In other words, if a point $\left(x_{m}, f\left(x_{m}\right)\right)$ is the end of the climb, we are looking for the beginning point $(x, f(x))$ for which the difficulty index of the road is the greatest. Obviously, $x \in\left[0, x_{m}\right)$.

According to (1) for the points $(x, f(x))$ and $\left(x_{m}, f\left(x_{m}\right)\right)$ we can introduce its slope as a function

$$
m(x)=\frac{f\left(x_{m}\right)-f(x)}{x_{m}-x}
$$

where

$$
x \in\left[0, x_{m}\right) .
$$

Similarly, from (2) we can describe the difficulty index as a function $t$ defined as

$$
t(x)=\left(\frac{f\left(x_{m}\right)-f(x)}{x_{m}-x}\right)^{2}\left(x_{m}-x\right)=\frac{\left(f\left(x_{m}\right)-f(x)\right)^{2}}{x_{m}-x}
$$

where

$$
x \in\left[0, x_{m}\right) .
$$

We are looking for the maximum of the function $t$.

\section{Calculations}

From (4) we may describe a more general case, where $t$ is defined as

$$
t(x)=\left(\frac{f\left(x_{m}\right)-f(x)}{x_{m}-x}\right)^{p}\left(x_{m}-x\right)=\frac{\left(f\left(x_{m}\right)-f(x)\right)^{p}}{\left(x_{m}-x\right)^{p-1}}
$$


where

$$
x \in\left[0, x_{m}\right) .
$$

Theorem 1. Let $f:\left[0, x_{m}\right] \rightarrow \mathbb{R}$ be continuous and differentiable. If $x \in\left[0, x_{m}\right)$ is an extreme point of the function $t:\left[0, x_{m}\right) \rightarrow \mathbb{R}$ given by (5) then $f(x)=f\left(x_{m}\right)$ or $f^{\prime}(x)=\frac{p-1}{p} \frac{f\left(x_{m}\right)-f(x)}{x_{m}-x}$.

Proof. Applying the differentiability of $f$ at $x \in\left[0, x_{m}\right]$ we easily get the following identities:

$$
\begin{aligned}
& t^{\prime(x)}=\left[\frac{\left(f\left(x_{m}\right)-f(x)\right)^{p}}{\left(x_{m}-x\right)^{p-1}}\right]^{\prime}= \\
& =\frac{\left[\left(f\left(x_{m}\right)-f(x)\right)^{p}\right]^{\prime}\left(x_{m}-x\right)^{p-1}-\left(f\left(x_{m}\right)-f(x)\right)^{p}\left[\left(x_{m}-x\right)^{p-1}\right]^{\prime}}{\left(x_{m}-x\right)^{2(p-1)}}= \\
& =\frac{p\left(f\left(x_{m}\right)-f(x)\right)^{p}\left(-f^{\prime}(x)\right)\left(x_{m}-x\right)^{p-1}+(p-1)\left(x_{m}-x\right)^{p-2}}{\left(x_{m}-x\right)^{2(p-1)}}= \\
& =\frac{\left(f\left(x_{m}\right)-f(x)\right)^{p-1}\left(x_{m}-x\right)^{p-2}\left[p\left(-f^{\prime}(x)\right)\left(x_{m}-x\right)+(p-1)\left(f\left(x_{m}\right)-f(x)\right)\right]}{\left(x_{m}-x\right)^{2(p-1)}}= \\
& =\frac{\left(f\left(x_{m}\right)-f(x)\right)^{p-1}\left[-p f^{\prime}(x)\left(x_{m}-x\right)+(p-1)\left(f\left(x_{m}\right)-f(x)\right)\right]}{\left(x_{m}-x\right)^{p}} .
\end{aligned}
$$

Since a necessary condition for $x$ to be an extreme point of $t$ is $t^{\prime}(x)=0$, we get

$$
f\left(x_{m}\right)-f(x)=0
$$

or

$$
-p f^{\prime}(x)\left(x_{m}-x\right)+(p-1)\left(f\left(x_{m}\right)-f(x)\right)
$$

therefore

$$
f\left(x_{m}\right)=f(x)
$$

or

$$
f^{\prime}(x)=\frac{(p-1)\left(f\left(x_{m}\right)-f(x)\right)}{p\left(x_{m}-x\right)} .
$$

The case (6) is not useful in our consideration (in this case $t(x)=0$ ), so let us consider the case (7). From (3) and (7) we get

$$
f^{\prime}(x)=\frac{p-1}{p} m(x) .
$$

It is an interesting observation. The value $f^{\prime}(x)$ is a local slope of the function $f$ in the point $x$. Thus, there is the following remark. 
Remark. An optimal beginning point of the road is in the point where the local slope equals the average slope of the road from that point to the peak multiplied by $\frac{p-1}{p}$.

For $p=2$ difficulty index is defined in (4) and an optimal beginning point fulfils the necessary condition

$$
f^{\prime}(x)=\frac{1}{2} m(x)
$$

\section{Graphic interpretation}

Figure $3 \mathrm{a}$ shows an altitude profile of the road described by the function $f(x)=-50 \cos \left(\frac{\pi x}{1000}\right)+300$. In Figure $3 b$ the solid line presents the function $f^{\prime}(x)=\frac{\pi}{20} x \sin \left(\frac{\pi x}{1000}\right)$. It is a local slope in each point $x$, in [3] graph like this is called a gradient profile. On the same graph, the dotted line presents the average slope defined by the function (3) and the dashed line presents a half of this function.

Figure 3c shows the difficulty index defined by (4). From Figures $3 a$ and $3 c$, we can read that difficulty index attains its maximum in the same point where the local slope equals a half of the average slope.

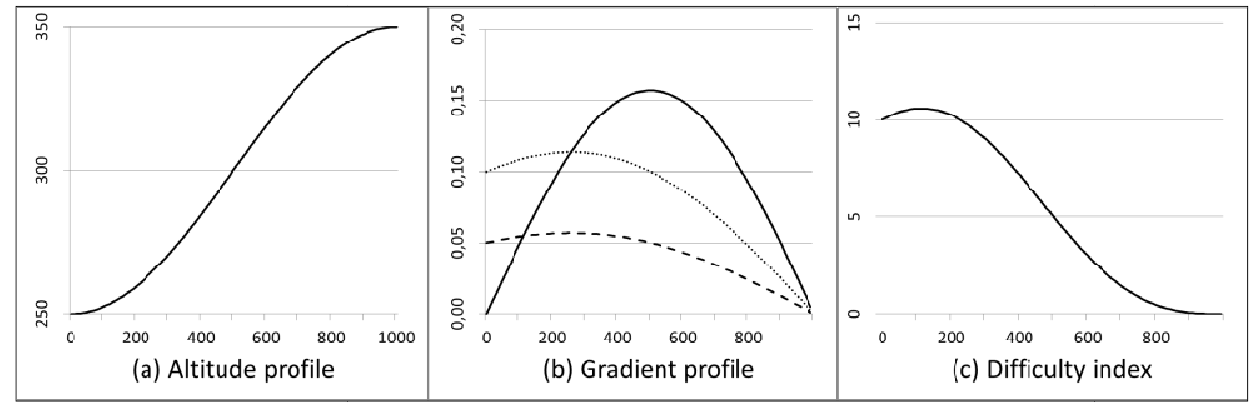

Fig. 3. Graphs relevant to the profile defined by $f(x)=-50 \cos \left(\frac{\pi x}{1000}\right)+300$

As was mentioned in Section 2, in practical cases the formula of $f(x)$ is unknown. There is usually only an altitude profile read available from measurements or a numerical terrain model. Using the altitude profile is possible to, with some accuracy, draw a gradient profile and graph on the average slope and this way estimate a point where the difficulty index is the greatest.

Figure 4 is similar to Figure 3, but presents a more complex road profile. In this case, the climb contains a little downhill inside. The local slope of this road equals a half of the average slope in three points. In each of them, the difficulty index attains its local extremum. 


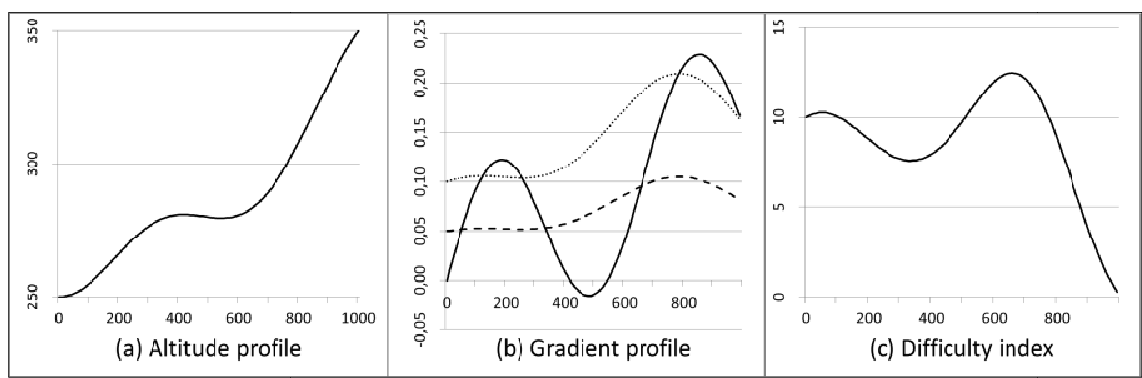

Fig. 4. Graphs relevant to the profile defined by $f(x)=-10 \cos \left(\frac{3 \pi x}{1000}\right)+\frac{8}{10^{5}} x^{2}+260$

\section{A special case of the road}

We can ask the following question: is there any road profile, that no matter which point we choose as the beginning point, the difficulty index is the same? It would have to be a function that in each point $t(x)$ is the same. Hence, from (4) we have:

$$
\frac{\left(f\left(x_{m}\right)-f(x)\right)^{2}}{x_{m}-x}=C, x \in\left[0, x_{m}\right)
$$

where $C$ is a constant. Multiplying both sides of (10) we have

$$
\left(f\left(x_{m}\right)-f(x)\right)^{2}=C\left(x_{m}-x\right)
$$

therefore

$$
f\left(x_{m}\right)-f(x)=\sqrt{C\left(x_{m}-x\right)}, x \in\left[0, x_{m}\right)
$$

$\sqrt{C}$ is just another constant, so without any loss of generality, (11) can be written as

$$
f(x)=f\left(x_{m}\right)-C \sqrt{x_{m}-x}, x \in\left[0, x_{m}\right)
$$

Example of this function is given in Figure 5.

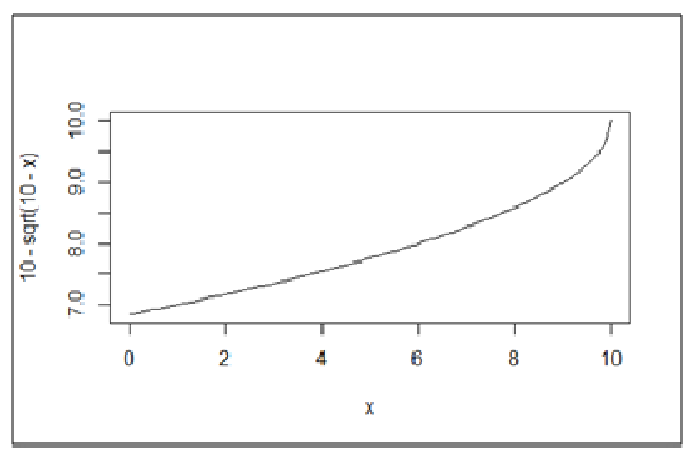

Fig. 5. The graph of the function $f(x)=10-\sqrt{10-x}$ 


\section{Conclusions}

If the difficulty index is defined by $t=m^{p} r$, an optimal place to establish the beginning of the climb is in one of the points where the local slope equals the average slope of the road from that point to the peak multiplied by $\frac{p-1}{p}$.

The simplest variant is $p=2$. In this case, the difficulty index is defined by $t=m^{2} r$ and an optimal point to set the beginning of the climb is in one of the points, where the local slope equals a half of the average slope from that point to the end of the climb.

The number of these points may be greater than 1, in some cases, presented in section 5 , this number is infinite.

\section{Acknowledgements}

The author wishes to express his thanks to Prof. Janusz Matkowski for his helpful suggestions during the preparation of this paper.

\section{References}

[1] Padilla, S., Mujika, I., Santisteban, J., Impellizzeri, F., \& José, G.J. (2008). Exercise intensity and load during uphill cycling in professional 3-week races. European Journal of Applied Physiology, 102(4), 431-8. DOI: 10.1007/s00421-007-0602-9.

[2] Wolf, S., Bertschinger, R., \& Saupe, D. (2016). Road cycling climbs made speedier by personalized pacing strategies. Proceedings of the 4th International Congress on Sport Sciences Research and Technology Support. 109-114. DOI: 10.5220/0006080001090114.

[3] Dahmen, T., Wolf, S., \& Saupe, D. (2012). Applications of mathematical models of road cycling. IFAC Proceedings, 45(2), 804-809. DOI: 10.3182/20120215-3-AT-3016.00142.

[4] El Helou, N., Berthelot, G., Thibault, V., Tafflet, M., Nassif, F., Campion, F., Hermine, O., \& Toussaint, J.F. (2010). Tour de France, Giro, Vuelta, and classic European races show a unique progression of road cycling speed in the last 20 years. Journal of Sports Sciences, 28(7), 789-796, DOI: $10.1080 / 02640411003739654$.

[5] How Are Climbs Categorised? (2015, July 15). Retrieved from http://inrng.com/2015/07/tourclimbs-category-formula/. 